\title{
SMOOTH AND ROUGH PLATINUM DEPOSITS RESULTING FROM THE ELECTROREDUCTION OF HYDROUS OXIDE PLATINUM OVERLAYERS A MECHANISTIC APPROACH
}

\author{
A. F. Bot t.an, A. M. Castro luna, A. Visintin, R. C. Salvarezza and A. J. Arvia \\ Instituto de Investigaciones Fisicoquimicas Téricas y Aplicadas (INIFTA), Faculad de Ciencias \\ Exactas, Universidad Nacional de La Plata, Sucursal 4, Casilla de Correo 16. (1900) La Plata, \\ Argentina
}

(Receited 16 March 1988; in revised form 10 May 1988)

\begin{abstract}
A bstract--The clectroreduction of thick platinum oxides accumulated by the application of a fast square wave perturbing potential in $0.5 \mathrm{M} \mathrm{H}_{2} \mathrm{SO}_{4}$ was studied by using potentiodynamic and potentiostatic techniques complemented with scanning electron miscroscopy. The electroreduction process can be unambigously explained through an instantaneous nucleation and 3-D growth of right circular cones under charge transfer control where diffusion of reacting species to the growing centres is essential for further expansion. The growth mode of the Pt crystallites either smooth or rough depends on the electroreduction conditions. The rough/smooth modes of growth result from the influence of the electric field on the transport of particles in the reaction region and on the supersaturation concentration operating during the electrocrystallization process.
\end{abstract}

\section{NOMENCLATURE}

$E$

E.

$E_{c}$

$E_{1}$

$E_{1}$
$E_{\mathrm{u}}$

$E_{r}$

$E_{\text {s, u }}$

$E_{\mathrm{x}, \mathrm{c}}$

$E_{x}$

$f^{\prime}$

$I_{M}$

$I_{\mathrm{m}}$

$j(t)$

$k_{1}$

$k_{2}$

$M$

$N_{4}$

$q$

R RSWPS

$\tau_{1}$

$\tau_{\mathrm{u}}$

id

$t_{\mathrm{M}}$

$t_{m}$

EA 33:12-D

applied potential inner $\mathrm{PtO}$ monolayer layer reversible potential sweep voltammetry tial sweep voltammetry

potential of zero charge

RSWPS frequency

current intensity apparent electrode arca the electrode surface to the electrode surface

number of nucleation centers

oxide charge density

density of the oxide phase

\section{INTRODUCTION}

potential step valuc for clectroreduction of the

potential step value for electroreduction oxide

lower potential limit in the RSWPS treatment upper potential limit in the RSWPS treatment

anodic switching potential in triangular potential

cathodic switching potential in triangular poten-

maximum current attained during the electroreduction current transient at $E_{\mathrm{c}}$

minimum current attained during the electroreduction current transient at $E_{\mathrm{c}}$ instantaneous current density referred to the

metal overlayer growth rate constant parallel to

metal overlayer growth rate constant orthogonal

molecular weight of the oxide phase

roughness factor as defined in the text

repetitive square wave potential signal

half period corresponding to $E_{1}$

half period corresponding to $E_{\mathrm{u}}$

duration of the RSWPS treatment

duration of the potential step at $E_{\mathrm{n}}$

time corresponding to the appearance of $I_{M}$

time corresponding to the appearance of $I_{m}$

The clectrode surface morphology and roughness are of utmost importance in electrocatalysis. These electrode characteristics in the case of metals can be handled by carrying out different procedures under established conditions such as chemical or electrochemical reduction of oxides, metal electrodeposition from soluble salts and metal dispersion on porous materials[1]. Although most of those procedures as applied to technical electrochemistry are essentially based upon empirical data, according to recent results the preparation of metal surfaces from oxide layers electroreduction offers a ncw perspective to investigate the relationship between the type of oxide layer, its clcctroreduction conditions and the morphology and roughness of the resulting electroreduced metal overlayer, and to interpret it in terms of fundamental electrochemistry.

It is well known that hydrous oxide multilayers can be formed on platinum electrodes by applying severe anodic polarization conditions by using $d c$ and $a c$ biased with $d c$ [2-9]. Another type of anodizing procedures was also developed to accumulate platinum metal oxide layers by using square wave perturbing potentials at frequencies of a few $\mathrm{kHz}$ thereabouts and potential windows ranging from 1.5 to $2.5 \mathrm{~V}[10-12]$. According to voltammetric, potentiostatic and galvanostatic electroreduction results and XPS data of relatively thick platinum oxides layers obtained in acid solution, those multilayers consist of different types of oxides $[13,14]$. However, the morphology, chemical structure and composition of these oxide layers depend strongly on the preparation conditions. In this respect, it appears that the water content of the 
oxide layer determines to a large extent their electrochemical behaviour, particularly in the metal surface morphology resulting through their electroreduction [10].

Various mechanisms have been advanced to account for the electroreduction of platinum oxide layers. Thus, on the basis that the structure of thick platinum oxide layers involves the contributions of an inner layer and an outer layer, it was concluded that the electroreduction of the inner layer follows a consecutive mechanism involving two electron transfer steps, the second one being rate determining, whereas the electroreduction of the outer layer undergoes through a place exchange mechanism[7]. Otherwise, it has been reportcd that the electroreduction rate of the platinum oxide multilayer formed under a constant potential is greater than its electroformation rate. This difference allowed us to conclude that the electroreduction reaction follows a proton-electron transfer mechanism, and that the structure for the platinum oxide layer consists of the thick platinum oxide layer occupying the intermediate region between the metal surface and an oxide monolayer in contact with the solution[7].

Finally, as concluded from potentiodynamic data, the electroreduction of the platinum oxide multilayer apparently takes place under ohmic control[15]. Therefore, the kinctic information available at present is not coincident ard furthermore, it furnishes no correlation between the electroreduction mechanism and surface roughness features of the resulting platinum overlayer.

This paper describes the kinetics of the electroreduction of platinum oxide layers which have been formed under a fast periodic square wave potential (RSWPS) treatment[12]. The electroreduction process is investigated by using potentiodynamic and potentiostatic techniques complemented by scanning electron microscopy. The present data unambiguously support a common electroreduction mechanism involving transport processes at the oxide layer level and a metal ion surface reaction under nucleation and growth kinetics. The proposed reaction model accounts for the potentiostatic current transients and explains some aspects of the influence of the electroreduction conditions on the surface roughness features of the resulting platinum overlayers.

\section{EXPERIMENTAL}

Two types of platinum working clectrodes were used, namcly polycrystalline (pc) platinum wircs (Johnson Matthey Chemical Co. 99.999\% purity) of 4-6 mm length and $0.5 \mathrm{~mm}$ dia and polyfacetted single crystal (pfsc) platinum elcctrodes. The latter were prepared as described in [16]. Prior to each run, the $p c$ platinum electrodes were electropolished in a saturated $\mathrm{CaCl}_{2}$ solution with a slight excess of $\mathrm{HCl}$ by applying $a c$ ( $50 \mathrm{~Hz}, 10-15 \mathrm{~V})$, then repeatedly rinsed with triply distilled water, and finally, immersed for $1 \mathrm{~h}$ in the electrolyte solution used in the cell $\left(0.5 \mathrm{M} \mathrm{H}_{2} \mathrm{SO}_{4}\right)$ at $25^{\circ} \mathrm{C}$. A large platinized platinum plate $\left(10 \mathrm{~cm}^{2}\right)$ was used as counterelectrode and it was placed directly into the main body of the cell to minimize the ohmic drop between the working elec- trode and the counterelectrode. The large counter electrode/working electrode surface area ratio and the short duration of each experiment eliminate any interference of products from the counter electrode coming into the working electrode region. A hydrogen reference electrode in the same acid solution connected to the coll through a I uggin-Haber capillary tip was employed.

Each experiment consisted of the following stages: (i) evaluation of the initial area of the working electrode through the voltammetric $O$ electrodesorption charge resulting at $0.1 \mathrm{~V} \mathrm{~s}^{-1}$ between $E_{\mathrm{s} . \mathrm{c}}=0.05 \mathrm{~V}$ and $E_{\mathrm{s}, \mathrm{a}}=1.40 \mathrm{~V}$ by taking for the $\mathrm{O}$ monolayer charge density $0.420 \mathrm{mC} \mathrm{cm}^{2}$ [17]; (ii) accumulation of the thick hydrous platinum oxide layer (henceforth denoted as the oxide layer). For this purpose the working electrode was subjected to an asymmetric RSWPS treatment at the frequency $f(5<j<1 \mathrm{kHz})$ between $E_{\mathrm{u}}=1.50 \mathrm{~V}$ and $E_{\mathrm{s}}=0.05 \mathrm{~V}$, during the time $t$, $(15<t<600 \mathrm{~s})$. The thickness of the oxide layer depended on $t$. The following optimal conditions were employed $E_{1}=0.05 \mathrm{~V} ; E_{\mathrm{u}}=1.50 \mathrm{~V} ; f=1 \mathrm{kHz}, \tau_{\mathrm{u}}$ $=923 \mu \mathrm{s}, \quad \tau_{1}=77 \mu \mathrm{s}[12]$ and $15<t<600 \mathrm{~s}$. Subsequently, the platinum oxide layer was kept at $E_{u}$ for $10 \mathrm{~s}$ to obtain a steady state for the current. (iii) Electroreduction of the oxide layer by using either a linear potential sean from $E_{\mathrm{u}}$ downwards at a preset swecp ratc, $v$, comprised in the $0.001-0.1 \mathrm{~V} \mathrm{~s}^{-1}$ range to reach $0.05 \mathrm{~V}$ or a potential step $E_{\mathrm{c}}$ in the $0.0-0.5 \mathrm{~V}$ range. The potentiostatic electroreduction was preceded by a step to $F_{\mathrm{a}}=0.62 \mathrm{~V}$, for $t_{\mathrm{a}}=15 \mathrm{~s}$, 10 electroreduce the platinum monoxide layer in contact with the metal. The oxide layer was completely electroreduced in the $0.0-0.5 \mathrm{~V}$ range. (iv) The electrochemical behaviour of the electroreduced platinum surfaces was checked through conventional voltammetry at $0.1 \mathrm{Vs}^{-1}$ in $0.5 \mathrm{M} \mathrm{H}_{2} \mathrm{SO}_{4}$ between $E_{\mathrm{s}, \mathrm{c}}-0.05 \mathrm{~V}$ and $E_{\mathrm{s}, \mathrm{d}}-1.45 \mathrm{~V}$ at $25^{\circ} \mathrm{C}$. The ratio between the $O$ electroreduction charge before and after the electrode treatment was used as the measurement of the electrode roughness $(R)$ of the platinum overlayer resulting from the oxide layer electroreduction. SEM micrographs of these overlayers werc systematically taken with a Philips 500 scanning electron microscope.

\section{RESULTS}

\section{Voltammetric data}

The complex current-potential display of the electroreduction process of a platinum oxide layer which has becn accumulated through the RSWPS treatment during $l=3$ min either on pc or pfsc platinum, and subsequently potentiodynamically electroreduced at $v=10^{-3} \mathrm{~V} \mathrm{~s}^{-1}$ from $E_{\mathrm{s}, \mathrm{a}}=1.5 \mathrm{~V}$ to $\boldsymbol{E}_{\mathrm{s}, \mathrm{c}}=0.0 \mathrm{~V}$, shows up a small peak (I) in the $0.60-0.75 \mathrm{~V}$ range which can be assigned to the electroreduction of the $O$ adatom monolayer $\left(0.420 \mathrm{mC} \mathrm{cm}{ }^{2}\right)$ followed hy a rather asymmetric peak (II) at $0.50 \mathrm{~V}$, involving a larger charge density, $4-0.4 \mathrm{C} \mathrm{cm}^{-2}$ (Fig. I, plot a) with a hump at the positive potential side. Peak I can be more clearly seen as $t$ is increased and $t$ is decreased (Fig. 1, plot b). Its peak potential shifts negatively as $t$ is increased (Fig. 1, plot c). At the end of the electroreduction scan and the beginning of the reverse scan a 


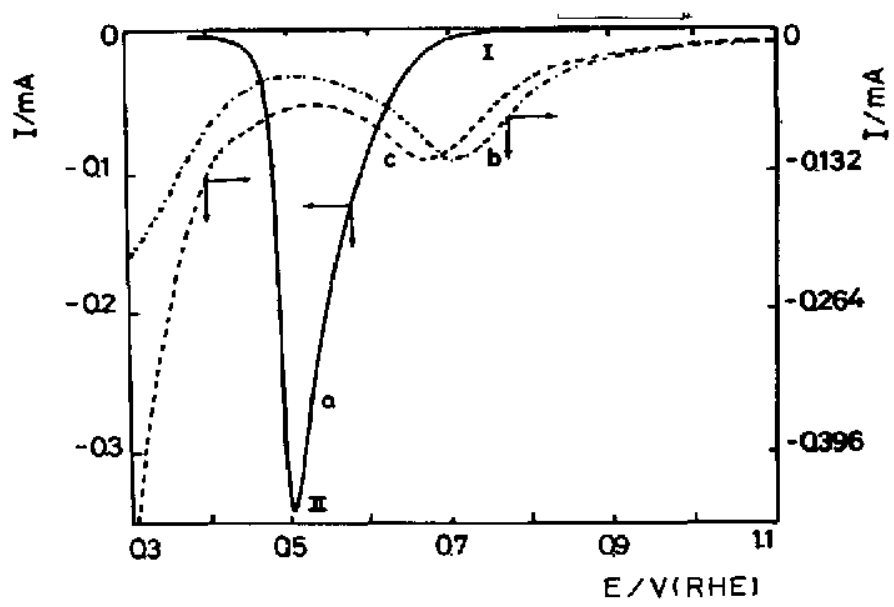

Fig. 1. E-I profiles recorded for the electroreduction of the platinum oxides in $0.5 \mathrm{M} \mathrm{H}_{2} \mathrm{SO}_{4},(-) v=1$ $\times 10^{-3} \mathrm{~V} \mathrm{~s}^{-1}, t=3 \mathrm{~min} ;(\cdot-\cdot) v=0.1 \mathrm{~V} \mathrm{~s}^{-1}, t=10 \mathrm{~s} ;(----) v=0.1 \mathrm{Vs}^{-1}, t=30 \mathrm{~s}$.

clear hysteresis loop can be observed which extends within the potential region of peak II (Fig. 2). The reaction associated with the electroreduction of the gross amount of accumulated oxide (hydrous platinum oxide) (peak II) is definitely responsible for the roughness features of the reformed $\mathrm{Pt}$ overlayer. However, at first sight it is surprising that for starting oxide layers prepared exactly under the same conditions the roughness features of the $\mathrm{Pt}$ overlayer become notoriously different according to the characteristics of the electroreduction process. For instance, the value of $R$ resulting from the potentiodynamic electroreduction within the same potential window, varies from 47 for $v=0.001 \mathrm{Vs}^{-1}$ to 12 for $v=0.1 \mathrm{Vs}^{-1}$ (Fig. 3). Likewise, the influence of $v$ also appears in the electrocatalytic properties of the resulting Pt overlayers, as derived from the change in the relative height of the $H$ adatom current peaks recorded in the $0.05-0.6 \mathrm{~V}$ range. Thus, for the $\mathrm{Pt}$ overlayers obtained from electroduction at $v>0.05 \mathrm{Vs}^{-1}$ the heights of the electroadsorption-electrodesorption peaks for strongly bound $\mathbf{H}$ adatoms increase as compared to weakly bound $\mathrm{H}$ adatoms (Fig. 4), whereas the opposite effect shows up for those Pt overlayers resulting from the electroreduction at $v<10^{-3} \mathrm{Vs}^{-1}$. These results indicate that not only $R$ depends on the scan rate of the electroreduction process, but the scan rate also determines the development of a certain preferred crystallographic orientation at the $\mathrm{Pt}$ overlayer.

\section{Potentiostatic current transients}

The oxide layers accumulated either on pc or pfsc platinum through the RSWPS treatment during different $t$ were subsequently electroreduced by stepping the potential from $E_{\mathrm{u}}=1.5 \mathrm{~V}$ to $E_{\mathrm{c}}$ in $0.50 \leqslant E_{c} \leqslant 0.0 \mathrm{~V}$. For this purpose the potentiodynamic electroreduction of the oxide layer was preceded by a $10 \mathrm{~s}$ potential holding at $E_{\mathrm{u}}=1.5 \mathrm{~V}$, later stepped to $E_{\mathrm{a}}=0.62 \mathrm{~V}$ for $15 \mathrm{~s}$ to electroreduce exclusively the $O$ adatom monolayer (Reaction $\mathrm{I}$ ) and immediately afterwards stepped to $E_{\mathrm{c}}\left(0.0<E_{\mathrm{c}}<0.50 \mathrm{~V}\right)$ to electroreduce the gross remaining hydrous portion of the oxide layer (Reaction II). It should be noted that the potentiostatic electrorcductions at potentials lower than about $0.4 \mathrm{~V}$ a charge contribution of the $H$ adatom electroadsorption appears which decreases approximately linearly with $E_{\mathrm{c}}$. This contribution reaches the maximum value $210 \mu \mathrm{C} \mathrm{cm}^{-2}$ for $E_{\mathrm{c}} \cong 0 \mathrm{~V}$. However, in the present case the $H$ adatom charge contribution to the overall electroreduction process is always $<0.1 \%$ as the oxide charge involved is about $350 \mathrm{mC} \mathrm{cm}^{-2}$. The current transients associated with Reaction $I$ always exhibit a monotonous decay and in this case the charge density resulting from integration is always smaller than $0.420 \mathrm{mC} \mathrm{cm}^{-2}$, the charge density expected for one monolayer of $\mathrm{O}$ containing surface species on $P t[17]$ with a value of $R$ equal to 1 . Obviously, neither the processes related to Reaction I nor those associated with the electrodissolution of $\mathrm{Pt}$ during the RSWPS treatment accounts for the change in $R$ accomplished under the electroreduction conditions already referred to above.

On the other hand, the current transients recorded for Reaction II depend strongly on both $E_{c}$ and $t$ (Fig. 5). Thus for $E_{\mathrm{c}}=0.37 \mathrm{~V}$ and $t=30 \mathrm{~s}$, the current transient firstly decreases, reaches a minimum value, $I_{\mathrm{m}}$, at the timc, $t_{\mathrm{m}}$ later increases sharply to attain a maximum value, $I_{\mathrm{M}}$ at the time $t_{\mathrm{M}}$, and finally, decreases markedly to reach a null current at $t=10 \mathrm{~s}$. The increase in $t$ from $30 \mathrm{~s}$ to 2 min results in a substantial increase in charge. In this case the current transient becomes broader, presumably because of a larger number of successive processes contributing to the overall electrochemical process. This effect can be scen, for instance, for $t>3 \mathrm{~min}$. For the latter the current transient exhibits two well-defined maxima, $I_{\mathrm{M}_{1}}$ at $t_{\mathrm{M}_{1}}$, and $I_{\mathrm{M}_{2}}$ at $t_{\mathrm{M}_{2}}$. The increase of $t$ produces only a slight change in $t_{M_{1}}$, but it shifts considerably $t_{M_{2}}$ towards longer times. Likewise, the charge associated with $I_{M_{1}}$ dccrcases as $t$ increases, whereas the opposite effect can be seen for the charge related to $I_{M_{2}}$. These facts suggest that a certain ageing of the oxide layer undergoes simultaneously as the RSWPS extends. This result is consistent with the fact that the potential of peak I shifts negatively as $t$ increases. It should be 


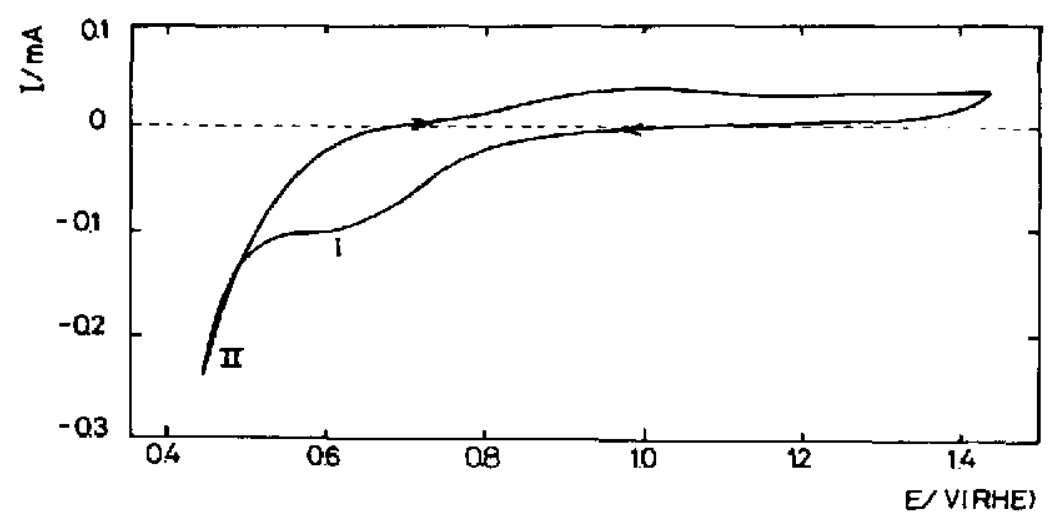

Fig. 2. E-1 prolile recorded at $r=0.05 \mathrm{~V} \mathrm{~s}^{-1}$ between $E_{4.4}=1.5 \mathrm{~V}$ and $E_{4 . \mathrm{c}}=0.32 \mathrm{~V}$ in $0.5 \mathrm{M} \mathrm{H}_{2} \mathrm{SO}_{4}$.

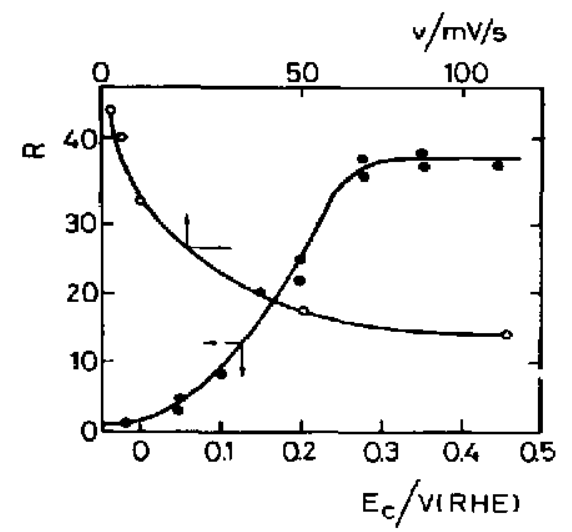

Fig. 3. $K$ vs $x$ plot, $t-3 \min (0)$ and $K x: F_{\mathrm{c}}$ plot, $t=3 \mathrm{~min}(\bullet)$ noticed, however, that the two current contributions appearing in the potentiostatic current transicnt correlate to both peak II and its hump, respectively, appearing in the voltammograms (Fig. 1, plot a).

When $E_{\mathrm{c}}$ increases both $I_{M_{1}}$ and $I_{M_{2}}$ increasc and $t_{M_{1}}$ and $t_{\mathbf{M}}$, decrease. Otherwise, when the oxide layer accumulated for a constant time $t$, is electroreduced at a constant potential $E_{\mathrm{c}}$ sct in the $0.25<E_{\mathrm{c}}<0.40 \mathrm{~V}$ range, the largest value of $R$ is attained. On the other hand, the value of $R$ becomes gradually smaller as $E_{\mathrm{c}}$ is fixed at potentials more negative than $0.25 \mathrm{~V}$ (Fig. 3). Finally, no increase in $\boldsymbol{K}$ can be observed for $E_{\mathrm{c}}=0.0 \mathrm{~V}$. For those runs made in the $0.25<E_{\mathrm{c}}<0.40 \mathrm{~V}$ range, the increase of $t$ results in a parabolic increase of $K$ with $E_{\mathrm{c}}$ (Fig. 6) reaching $R=60$ for $t=6 \mathrm{~min}$. The platinum overlayer obtained from the oxide layer electroreduction in the $0.45<E_{c}<0.35 \mathrm{~V}$ range exhibits no change as compared to the blank for the $H$ adalom voltammetric

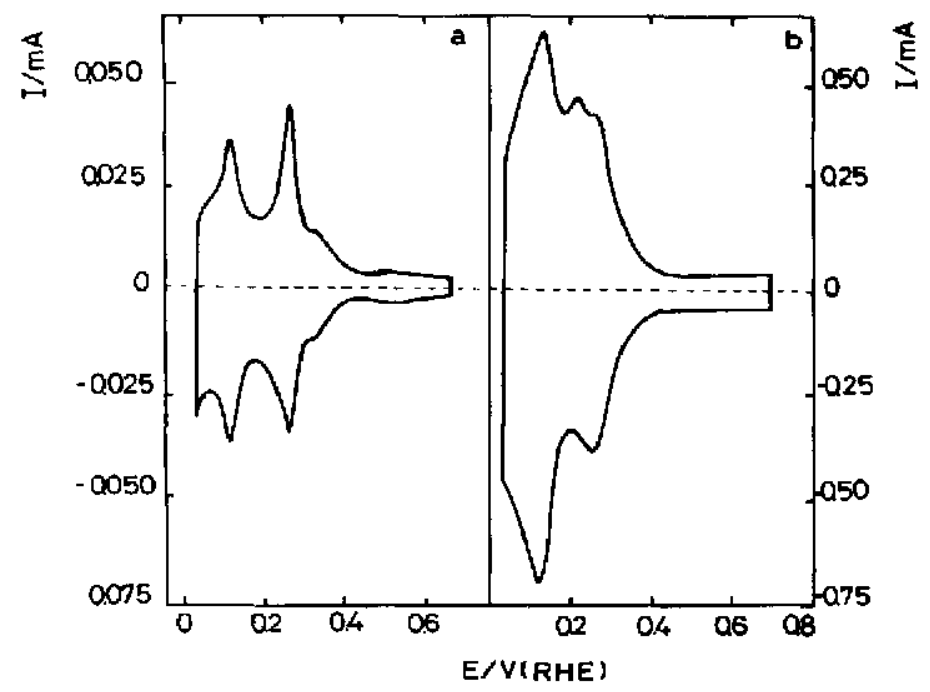

Fig. 4. $E-I$ profiles recorded at $v=0.10 \mathrm{~V} \mathrm{~s}{ }^{1}$ between $E_{\mathrm{B}, \mathrm{a}}=0.6 \mathrm{~V}$ and $E_{\mathrm{A}, \mathrm{c}}=0.05 \mathrm{~V}$ in $0.5 \mathrm{M} \mathrm{H}_{2} \mathrm{SO}_{4}$ after the electroreduction of the platinum oxides at (a) $v=0.1 \mathrm{Vs}^{-1} ;$; (b) $v=1 \times 10^{-3} \mathrm{~V} \mathrm{~s}^{-1}$. 


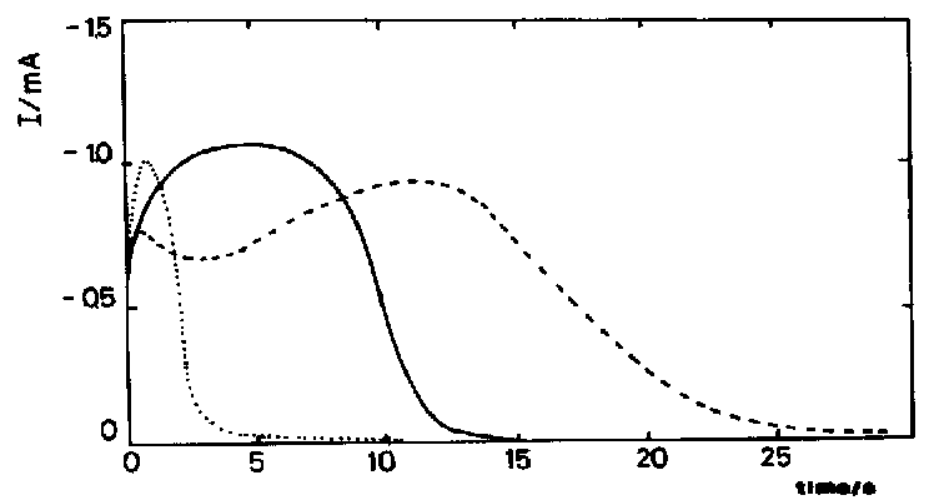

Fig. 5. Current transient at constant potential, $E_{\mathrm{c}}-0.35 \mathrm{~V}$ for $t=30 \mathrm{~s}(\cdots), t=2 \mathrm{~min}(\longrightarrow), t=5 \mathrm{~min}$ $(---)$.

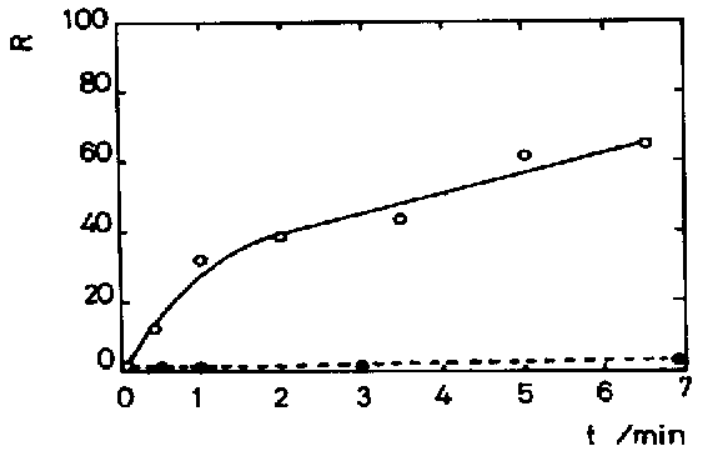

Fig. 6. $R$ vs $t$ plot. (ø) $E_{\mathrm{c}}=0.05 \mathrm{~V}$; (o) $E_{\mathrm{c}}=0.35 \mathrm{~V}$.

peaks. Conversely, for oxide layer electroreduced in the $0.35<E_{\mathrm{c}}<0.25 \mathrm{~V}$ range, the resulting platinum overlayer exhibits a predominant contribution of the voltammetric peaks assigned to weakly bound $H$ adatoms. Otherwise, the opposite trend can be observed for platinum overlayers resulting from the elctroreduction of the oxide layer in the $0.25<E_{\mathrm{c}}<0.0 \mathrm{~V}$ range.

\section{SEM observations}

The SEM micrographs of platinum overlayers resulting from the potentiostatic electroreduction of the oxide layers formed for $t=3 \mathrm{~min}$, change according to $E_{\mathrm{c}}$. Thus, when the electroreduction proceeds in the $0.35<E_{\mathrm{c}}<0.20 \mathrm{~V}$ range the surface morphology exhibits sticking spike-like units of ca $0.3 \mu \mathrm{m}$ average size dominating the surface, and a nearly uniform distribution of cavities. These morphological features are clustered and exhibit 3-D growth out of the starting platinum plane. The surface concentration of roughness features is approximately $10^{9} \mathrm{~cm}^{-2}$ in terms of spike-like units (Fig. 7). This surface morphology is typically related to large values of $R$. Otherwise, for $E_{\mathrm{c}}=0.0 \mathrm{~V}$, the surface morphology shows up compact pyramidal spikes with a density of about $10^{8}$ spikes $\mathrm{cm}^{-2}$ (Fig. 8). Obviously, small $R$ values result for this type of morphology.

\section{DISCUSSION}

Results indicate that surfacc properties such as real surface area and preferred crystallographic orientation of Pt overlayers obtained from the electroreduction of thick hydrous platinum oxide layers are strongly dependent on the history of the electroreduction process, that is, on either the linear potential sweep rate and potential window or potential step value. Furthermore the electrochemical data derived from the electroreduction of oxide layers accumulated under the relatively fast periodic potential $(f \cong 1-5 \mathrm{kHz})$ treatments with potential windows (their average potentials exceeding the equilibrium potential of the Pt-Pt(II) and Pt(II)-Pt(IV) redox couples[18]) favour an oxide layer structure made of an inner PtOH or PtO monolayer and a thick outer layer which involves a highly hydrous $\mathrm{Pt}(\mathrm{II})$ and Pt(IV) oxide species $[10,15]$. The electroreduction of these hydrous oxide layer can be described by considering those oxides as reactants and the incorporation of Pt ions into the metal ionic lattice to build up the platinum overlayer.

From the physical standpoint the overall process can be depicted as an electrochemically induced change from the oxide layer into the platinum overlayer. For the sake of simplicity such a process can be described in terms of two limiting possibilities. The first one considers that the initial state is the oxide layer with a density value approaching that of the aqueous electrolyte solution, and the final state comprises the platinum overlayer with the density value of bulk Pt. For such a reaction, in going from the initial to the final state one should expect the largest decrease in volume and correspondingly, the lowest roughness at the resulting void-free platinum overlayer. The second possibility arises when the reaction proceeds from the initial state to the final state without apparent density change. This means that the reaction proceeds under nearly a constant volume condition, so that the final state turns out to be a highly porous Pt structure with a large macroroughness.

The shape of the current transients and the voltammetric loop detected in the early stages of the electroreduction process (Figs 2 and 5 ) allow us to conclude 


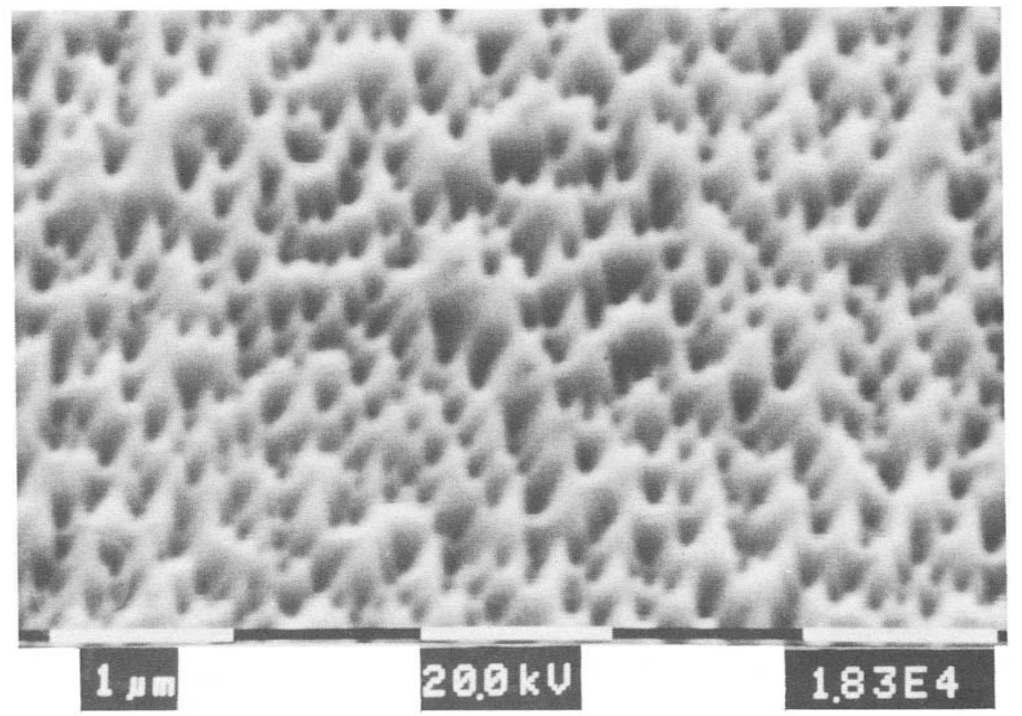

Fig. 7. SEM micrograph of the clectrode surface after the polentiostutic electroreduction of the platinum oxides at $E_{\mathrm{c}}=0.35 \mathrm{~V}: t=3 \mathrm{~min} ; R=40$.

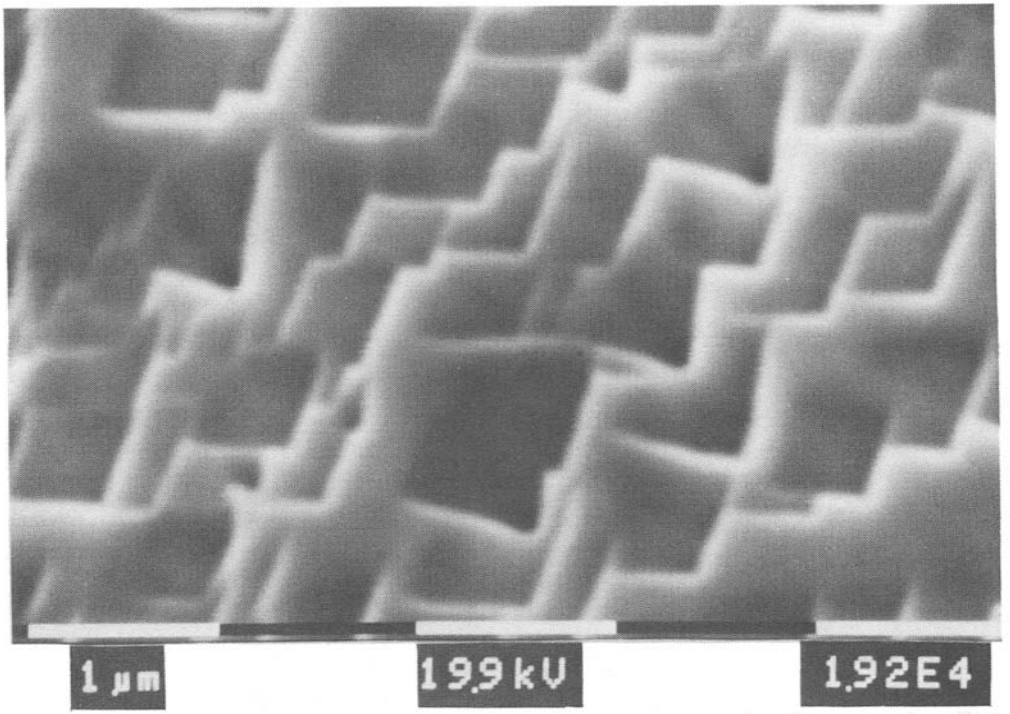

Fig. 8. StM micrograph of the electrode surface after the potentiostatic electroreduction of the platinum oxides at $E_{\mathrm{c}}=0.05 \mathrm{~V}, t=3 \mathrm{~min}, R-3$.

that the kinetics of the reaction follows the phenomenology of a nucleation and growth mechanism. Accordingly, as the oxide layer clcctroreduction proceeds at a potential more negative than the reversible potential of the Pt-Pt oxide redox couples, a critical supersaturation concentration of Pt species at the reaction interface must be reached to favour the nucleation and growth of $\mathrm{Pt}$ clusters in the innermost part of the oxide layer in contact with the metal surface, up to a distance compatible with a resonable high electron tunnelling probability.
In order to gain more information about the kinetics of the early stages of the oxide electroreduction process, particular attention has to be paid to the analysis of the current transients. The current transients comprising $t$ values shorter than $3 \mathrm{~min}$ exhibit a main current peak involving the electroreduction charge of the thick hydrous part of the oxide taycr. In contrast, for values of $t$ longer than 3 min the appearance of two peaks in the transients resulting from the electroreduction of the inner part of the oxide layer modified to some extent by the ageing effects, makes 
the interpretation in terms of relatively simple models more difficult.

The feature of the Pt layer as seen through the SEM micrographs arc consistent with a 3-D growth process. Nevertheless, simple nucleation and 3-D growth mcchanisms under charge transfer control imply a current transient where as $t \rightarrow \infty$ the current approaches a constant value, in contrast to the dependence of $I_{M}$ and $t_{\mathrm{M}}$ on $E_{\mathrm{c}}$ as derived from single peaked current transients. This apparent inconsistency, however, can be solved by considering that the oxide electroreduction process implies a nucleation and 3-D growth pyramids (Figs 7 and 8 ) under charge transfer control. The reaction takes place on a planar electrode where the diffusion of species from the electrode surface to the growing centres is essential for further expansion. Such a model has been successfully applied to the electrocrystallization of different anodic films $[19,20]$. Correspondingly, for an instantaneous nucleation and pyramids being right circular cones the current transient is represented by the equation [19]:

$$
\begin{array}{r}
j(t)=n F k_{1}\left[1.0-\exp \left(-\pi M^{2} k_{2}^{2} \frac{N_{0}}{\rho^{2}} t^{2}\right)\right] \\
\quad \times \exp \left(-\frac{\pi M^{2} k_{2}^{2} N_{0} t^{2}}{\rho^{2}}\right)= \\
=P_{1}\left[1.0-\exp \left(-P_{2} t^{2}\right)\right] \exp \left(-P_{2} t^{2}\right),
\end{array}
$$

where

$$
P_{1}=n F k_{1}
$$

and:

$$
P_{2}=\pi M^{2} k_{2}^{2} N_{0} i \rho^{2}
$$

and $k_{2}$ and $k_{1}$ are the growth rate constants parallel and orthogonal to the clectrode surface, respectively. $M$ and $\rho$ are the molecular weight and the density of the new phase, respectively and $N_{0}$ is the number of sites available for nucleation. The final exponential factor in Equation (1) takes into account the reduction of the growth rate caused by diffusion of the reacting species to growing sites (death). Accordingly, the depletion of the reacting species, results from the fact that there is a finite reservoir of material in the oxide layer. By using Equation (1) and the parameters assembled in Table 1 , the current transicnts can be satisfactorily reproduced (Figs 9 and 10) except for the slight deviation at short times, presumably due to the rather uncertain cstimation of the initial falling current which partially interferes the nucleation and growth process related to the electroreduction of the hydrous constituent of the oxide layer.

From the simulation parameters one can observe that the values of the rate constants $P_{1}$ and $P_{2}$ are

Table 1. Adjusting parameters used in current transient simulations for different values of $E_{u}$ and $t$

\begin{tabular}{lccc}
\hline$E_{\mathrm{u}} / \mathrm{V}$ & $t / \mathrm{min}$ & $P_{1} / \mathrm{mA} \mathrm{cm}^{-2}$ & $P_{2} / \mathrm{s}^{-2}$ \\
\hline 0.0 & 1 & 118.9 & 0.313 \\
0.0 & 3 & 146.8 & 0.162 \\
0.20 & 1 & 104.9 & 0.007 \\
0.27 & 3 & 69.9 & 0.0014 \\
\hline
\end{tabular}

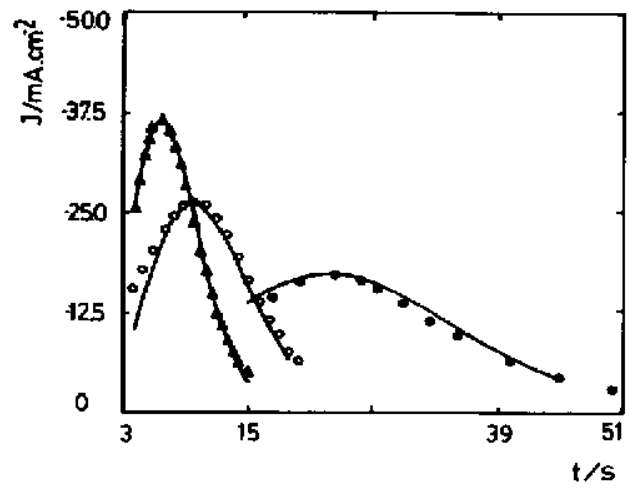

Fig. 9. Current transients at constant potential, $E_{\mathrm{c}}$ for the electroreduction of the platinum oxides. (A) $E_{\mathrm{c}}=0 \mathrm{~V}$, $t=3 \mathrm{~min}$; (O) $E_{\mathrm{c}}=0.2 \mathrm{~V}, t=1 \mathrm{~min}$; (๑) $E_{\mathrm{c}}=0.27 \mathrm{~V}, t=3 \mathrm{~min}$. Full lines: current transients calculaled by using Equation (1) and parameters assembled in Table 1. Apparent Pt electrode area: $0.08 \mathrm{~cm}^{2}$.

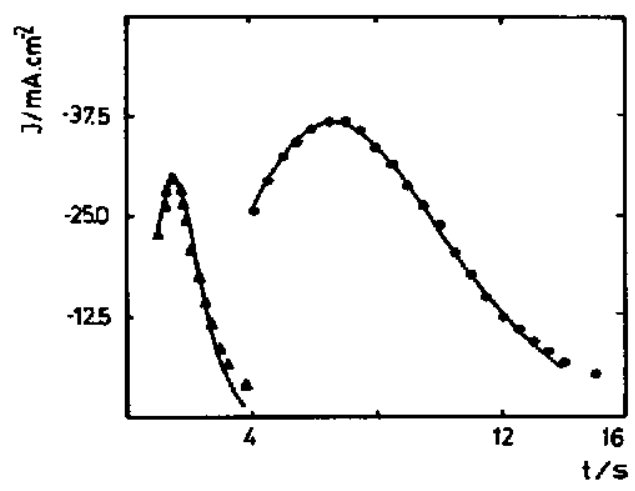

Fig. 10. Current transients at constant potential, $E_{\mathrm{r}}=0 \mathrm{~V}$, for the electroreduction of the platinum oxides $(\Delta) t=1 \mathrm{~min}$, (๑) $t=3 \mathrm{~min}$. Ful] lines: current transients calculated by using Equation (1) and parameters assembled in Table 1. Apparent Pt electrode area: $0.08 \mathrm{~cm}^{2}$.

nearly constant in the $0.100 .0 \mathrm{~V}$ range, although both increase in the $0.40-0.10 \mathrm{~V}$ range, reasonably fitting $\log P_{1}$ vs $E_{\mathrm{c}}$ and $\log P_{2}$ vs $E_{\mathrm{c}}$ linear plots for $E_{\mathrm{c}}>0.1 \mathrm{~V}$ (Fig, 11). The slopes of these plots are both approximately equal to $0.30 \mathrm{~V} \mathrm{dec}^{-1}$ (ie about $5 \mathrm{R} T / \mathrm{F}$ units). In principle, these slopes can be assimilated to Tafel slopes in stationary kinetics for the eletron trunsfer reactions proceeding either at the oxide layer or at the metal surface. Their relatively high values are, in principle, consistent with an electrochemical process strongly depressed through an electric field set-up either at the oxide layer or at the solution side due to a supercritical accumulation of cations at adjacent positions of the electrode and counter ions lying deeper into solution. The fact that $P_{2}$ decreases as $t$ increases favours the idea that the value of $P_{2}$ depends on $N_{0}$, the latter changing according to the oxide layer history and oxide charge density as seen for instance in Fig. 11, where two reasonably linear $\log \left(P_{2}^{1 / 2}\right)$ vs $E_{\mathrm{c}}$ plots are obtained for $q<20 \mathrm{mCcm}^{-2}$, and 


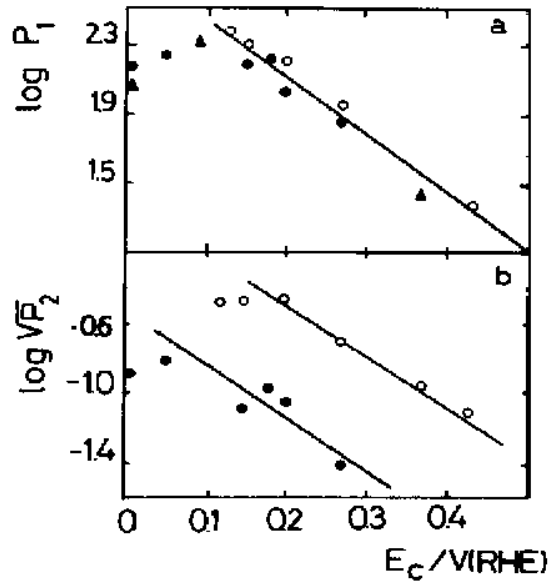

Fig. 11. $\log P_{1}$ ws $E_{\mathrm{c}}$ (a) and $\log \left(P_{2}^{1 / 2}\right)$ is $E_{\mathrm{c}}$ (b) for different oxide electroreduction charge density. ( $\Delta) q<10 \mathrm{mC} \mathrm{cm}^{-z}$; (O) $q>20 \mathrm{mC} \mathrm{cm}^{-2}$; (๑) $q<20 \mathrm{mC} \mathrm{cm}^{-2}$.

$q>20 \mathrm{mC} \cdot \mathrm{cm}^{-2}$, respectively. The influence of the oxide history on the kinetics of the electroreduction process is not fully understood at the present time and deserves further investigation.

The nucleation and growth model for the oxide layer electroreduction is obeyed over the entire potential range associated with that reaction. This conclusion, however, implics the limitation that the smooth and rough mode of growth of the Pt surface cannot be differentiated through the kinetic parameters derived from the clectrochcmical cxperiments. Nevertheless, a recent statistical-atomistic approach for the electroreduction of the oxide layers $[21]$ in the absence of specific adsorption shows that the value of $E_{\mathrm{c}}$ plays an important role during the electroreduction process in two aspects, as it influcnces the transport of the reacting particles from the oxide layer to the growing $P t$ centres and it also changes the supersaturation concentration of reacting particles which is proportional to the overvoltage defined as $\eta=E_{\mathrm{c}}-E_{\mathrm{r}}$. Let us first consider that $E_{c}$ is close to the zero charge potential $\left(E_{z}\right)$ of the metal substrate, the electric field effect on the transport of reacting particles can be neglected so that they travel practically at random before sticking to the growing Pt centres. Therefore, for low $\eta$ values, the collision rate of reacting particles to the growing Pt centres is small and is accompanicd by the formation of voids filled up with the solution constituents. Hence, when the electroreduction reaction proceeds under these conditions the overlayer growth involves a reaction approaching the constant volume condition. The topography of the Pt overlayer obscrved from the SEM micrographs (Fig. 7) is consistent with the preceding conclusions. In this cuse, the reacting volume can be estimated from the geometric electrode area times the average oxide layer thickncss (h). The latter can be roughly estimated from the relationship:

$$
h=\left(\begin{array}{c}
M \\
z F_{\rho}
\end{array}\right) q,
$$

by assuming that the initial layer consists of $\mathrm{PtO}_{2}$, so that by setting $t=1 \mathrm{~min}, \quad y=0.4 \mathrm{Ccm}^{-2}, M=$ $227.12 \mathrm{~g} \mathrm{~mol}^{-1}, \rho=10.2 \mathrm{~g} \mathrm{~cm}^{-3}$ and $z=4$, a value of $0.23 \mu \mathrm{m}$ for $h$ results. A nalogously, for the initial layer consisting of PtO, $M=211.05 \mathrm{~g} \mathrm{~mol}^{-1}, \quad \rho=$ $14.9 \mathrm{~g} \mathrm{~cm}^{-3}, z=2, h$ is close to $0.30 \mu \mathrm{m}$. Both values of $h$ are comparable to the average height of spike-type structure Pt overlayer formed in the potential range close to $E_{\mathrm{z}}$. Therefore, it can be concluded that as the phase change approaches the constant volume condition, the result is a Pt overlayer with a porous structure.

Let us now consider the second possibility, ie the applicd ficld assists the transport of reacting particles and in addition the value of $\eta$ is sufficiently large to assure a large supersaturation of reacting species. In this case, the rate of collision of reacting particles to the $\mathrm{Pt}$ centres becomes very fast and it is accompanied by a drastic change in volume resulting in $\mathrm{Pt}$ overlayer with an extremely low value of $R$. In this case the $\mathrm{Pt}$ ovcrlayer appears as a smooth surface with only a small number of spike-type structures (Fig. 7).

These conclusions are consistent with Monte Carlo simulation of growing metal crystallites with elongated structure at low supersaturation and growing metal crystallites with hemispherical structure at high supersaturation[22]. Hence a rough/smooth transition at a certain critical supersaturation for the growth mode of crystallites has received both experimental and theoretical support $[23,24]$.

Acknowledgments-This work was financially supported by the Consejo Nacional de Investigaciones Cjentificas y Técnicas and the Comisión de Investigaciones Cientificas de la Provincia de Buenos Aires. This work was also partially supported by the Regional Program for the Scientific and Technological Development of the Organization of the American States.

\section{REFERENCES}

1. K. Kinoshita and P. Stonehart, in Modern Aspects of Electrochemistry (Edited by J. O'M. Bockris and B. E. Conway), Vol. 12, Ch. 4, p. 183, Plenum Press, New York (1977).

2. J. P. Hoare, Electrochim. Acta 9, 276 (1964).

3. S. Gilman, J. electrnanal. Chem. 9, 276 (1965).

4. T. Bicgler, J. electrochem. Soc. 116,1138 (1969).

5. S. Shibata and P. Sumino, Electrochim. Acto 16, 1089 (1971).

6. S. Shibata, Electrochim. Acta 17, 395 (1972).

7. S. Shibata and P. Sumino. Electrochim. Acta 26, 517 (1981).

8. Yu. B. Vassilicv, V. S. Bagotzky and V. A. Gromyko, $J$. electroanal. Chem. 178, 247 (1984).

9. Yu. B. Vassiliev, V. S. Bagotzky and O. A. Khazova, J. electrocmal. Chem. 181, 219 (1984).

10. A. C. Chialvo, W. F. Triacia and A. J. Arvia, J. electrounal. Chem. 146, 93 (1983).

11. A. Visintin, W. E. Triaca and A. J. Arvia, J. electroanal. Chem. 221, 239 (1987).

12. A. Visintin, J. C. Canullo, W. E. Triaca and A. J. Arvia, J. electroanal. Chem. 239, 67 (1988).

13. M. Peuckert and H. lbach, Surf. Sci. 136, 319 (1984).

14. M. Peuckert, F. P. Coenen and H. P. Bonzel, Electrochim. Acta 29, 1305 (1984).

15. L. D. Burke and M. B. C. Rochc, J. electroanal. Chem. 164, 315 (1984).

16. J. C. Canullo, W. E. Triaca and A. J. Arvia, J. electroanal. Chem. 175, 337 (1984). 
17. R. Woods, Electroanalytical Chemistry (Editcd by A. J. Bard), Vol. 9, Ch. 1, p. 1, Dekker, New York (1976).

18. A. J. Bard, R. Parsons and J. Jordan (Editors), Standard Potentials in Aqueous Solutions, p. 353, Dekker, New York (1985).

19. R. D. Armstrong. M. Fleischmann and H. R. Thirsk, J. electroanal. Chem. 11, 208 (1966).

20. J. Gómez Becerra, R. C. Salvarezza and A. J. Arvia,
Electrochim. Acta 33, 1431 (1988).

21. E. Albano, H. O. Mártin und A. J. Arvia, in preparation. 22. A. Troyanov and. D. Kashchicv, J. Crystal Growth 78, 399 (1986).

23. D. Kashchiev, I. P. von der Eerden and C. von Leeuwen, J. Crystal Growth 40,47 (1977).

24. D. Kashchiev, J. Crystal Growth 40, 29 (1977). 